“C 2017 IEEE. Personal use of this material is permitted. Permission from IEEE must be obtained for all other uses, in any current or future media, including reprinting/republishing this material for advertising or promotional purposes, creating new collective works, for resale or redistribution to servers or lists, or reuse of any copyrighted component of this work in other works." 


\title{
A More Accurate Analog Voltage-Based Photovoltaic Maximum Power Point Tracking Technique
}

Mohammad R. Al-Soeidat, Student Member, IEEE School of

Electrical, Mechanical and

Mechatronic Systems, University of

Technology, Sydney, Australia mohammad.r.al-

soeidat@student.uts.edu.au

\author{
Dylan D-C. Lu, SMIEEE \\ School of Electrical, Mechanical and \\ Mechatronic Systems, \\ University of Technology, Sydney, \\ Australia \\ dylan.lu@uts.edu.au
}

\author{
Jianguo Zhu, SMIEEE \\ School of Electrical, Mechanical and \\ Mechatronic Systems, \\ University of Technology, Sydney, \\ Australia \\ yozhang@ieee.org
}

\begin{abstract}
In this paper, an analog voltage based maximum power point tracking (MPPT) algorithm for individual photovoltaic (PV) panel is proposed. The fixed voltage reference method is the simplest method for tracking, but it does not give good MPPT efficiency because the MPP voltage point changes at different solar insolation levels. A roughly linear slope is formed when connecting the MPP points measured from the highest isolation level to the lowest. Utilizing this characteristic, a bipolar junction transistor BJT is used to implement a variable voltage reference that improves the accuracy of the maximum power point voltage when the insolation changes. The proposed circuit is simple and easy to implement and it can easily track the maximum power point without the need of a digital controller or PID controller, so the cost and circuit complexity is reduced.
\end{abstract}

Keywords-component; maximum power point (MPP), solar energy, photovoltaic (PV), analog control, renewable energy, bipolar junction transistor (BJT), feedforward control.

\section{INTRODUCTION}

In recent years, using renewable energy such as wind, solar and fuel cell to generate electricity has been growing dramatically. Harvesting the maximum power form the photovoltaic (PV) module is carried out by inserting a power converter between the PV module and the load. This power converter operates at a different duty cycle (D) in order to match the output and input impedance[1]. For the PV module, it is necessary that the operation point of the power converter is set at the maximum power point (MPP) to harvest the maximum power for the (PV) Module [2] as shown in Fig. 1.

The solar insulation on the PV module could be affected by the clouds, planes and birds that are passing over the PV module. It could also be affected by the buildings and trees around PV module as well as the solar radiation angle. Thus, power generated from the PV module will change as it is shown in Fig. 2. As a result, a DC-DC power converter needs to be connected with a controller to sense the input/output voltage and current in order to generate the proper pulse width modulating (PWM) with different duty cycle to match the input and output impedances [3].
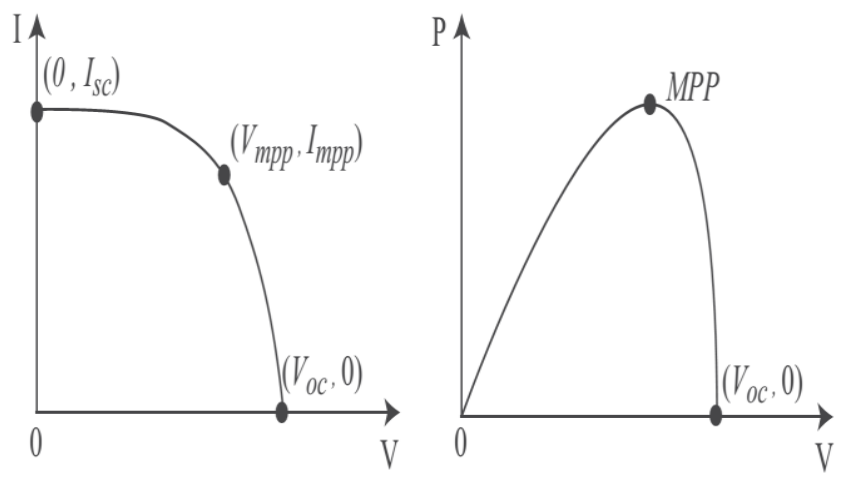

Fig. 1. Characteristic curves of a PV array.

In the last decades, a lot of maximum power point algorithms (MPPT) have been proposed such as fraction open circuit voltage (FOC), fraction short circuit current (FSC), incremental conduction (InC) and perturb and observe (P\&O) [4-7]. However, each algorithm has its pros and cons, such as the accuracy and required time of detecting the MPP. For example, FOC and FSC depend on measuring the open circuit voltage and short circuit current respectively. Thus, it is required to disconnect the PV module or the 
load every time that the radiation is changed so there are frequent and long power outages from the load perspective if the radiation changes rapidly. For P\&O and InC, the PV voltage oscillates around the maximum power point. There is also a need to use digital control as memory function is required so the cost of the system will be increased.

In this paper, a simple and cost effective MPPT analog algorithm is proposed for stand-alone system. The algorithm is voltagebased, and it depends on the characteristics of the PV module. The paper is organized as follows: In Section II, the proposed analog maximum power point algorithm will be examined. In Section III, the system setup will be discussed. The results are explained and shown in Sections IV, followed by the conclusion in Section V.
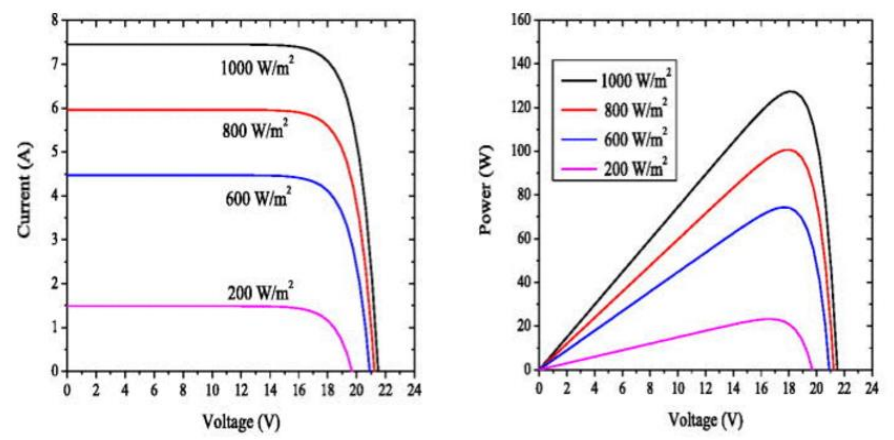

Fig. 2. Characteristic curves of a PV array.

\section{PROPOSED ANALOG MAXIMUM POWER POINT TRACKING ALGORITHMS}

\section{A. Propused Algorthim}

The proposed algorithm is a voltage-feedforward control [8]. It is easy to implement, and cost effective. In addition, it maximizes the potential of the PV module. The proposed algorithm is somewhat similar to the fraction open circuit voltage algorithm where the open circuit voltage has to be measured $\left(\mathrm{V}_{\mathrm{OC}}\right)$ to calculate the voltage at MPP $\left(\mathrm{V}_{\mathrm{MPP}}\right)$ according the below liner relationship[4]:

$$
V_{M P P}=k 1 * V_{O C}
$$

where $\mathrm{k}_{1}$ is a proportional constant, and it has a value between 0.71 and 0.78 depending on the characteristics of the PV module. However, in the proposed algorithm there is no need to disconnect the PV module to measure ( $\mathrm{V}_{\mathrm{OC}}$ ), so the close loop voltage feedforward can be used. As a result, the drawbacks of fractional open circuit voltage are solved.

As it is shown in Fig. 3, the maximum power points of PV module under different solar radiation are located on one line. Note that this line has a small slope. The idea of the proposed algorithm is to operate the power converter along with this line by using variable reference voltage. This reference voltage will be changed according to the solar radiation by using the bipolar junction transistor (BJT) that works on the active region on the feedforward loop.



Fig. 3. P-V curve and MPPT for different solar insulation. 


\section{B. BJT Transistor}

The bipolar junction transistor (BJT) transistor shown in Fig. 4 has four regions of operation which are cutoff, saturation, active and breakdown region. In the active region the collector current will change depending on the change of the base current [9]. BY apply Kirchhoff's voltage low (KVL) on the output close loop, its equation could be derived as below:

$$
V_{C}-I c R c-V_{C E}=0
$$

By solving above equation the load line and the characteristics curve of the BJT could be drown as it shown in Fig. 5. As a result, if the base current equals $\mathrm{I}_{\mathrm{B} 1}$ the operation point will be at point $A$ and the $\mathrm{V}_{\mathrm{CE}}$ will have a certain value. On the other hand, if the base current increases to become $\mathrm{I}_{\mathrm{B} 2}$, the operation point will move to point $\mathrm{B}$ and $\mathrm{V}_{\mathrm{CE}}$ will decrease and so on. In this case, when the base current of BJT can relate to the PV current, then it is possible to generate a variable reference voltage that matches the desired slope of voltage points for MPPT.

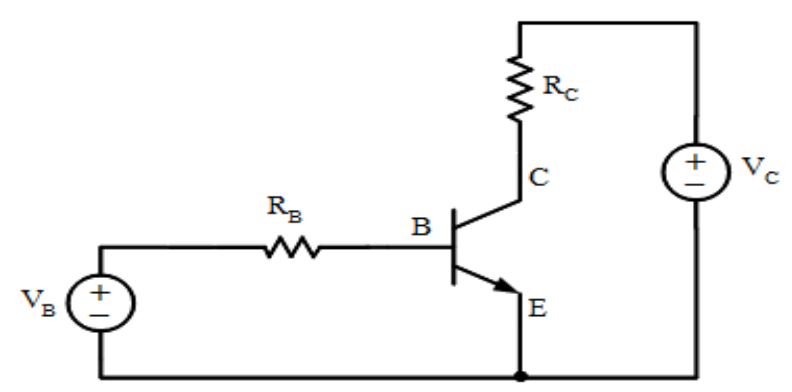

Fig. 4. BJT circuit .

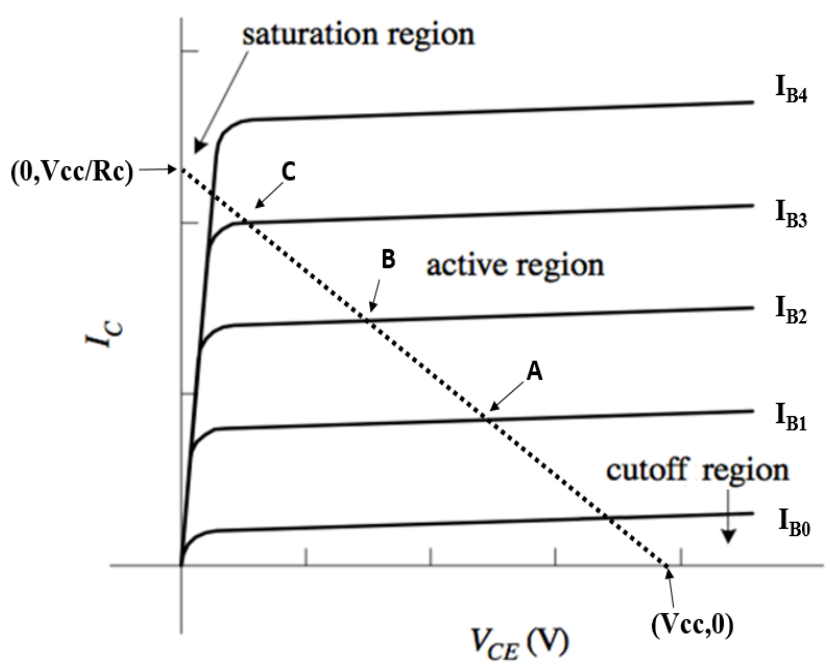

Fig. 5. BJT characteristics and the operation point.

\section{PWM signal gernating in analog control}

Fig. 6 shows PWM signal that is used in the proposed algorithm. Firstly, PWM signal needs to be generated from a function generator, so this signal will have a constant frequency, amplitude and duty cycle which are $10 \mathrm{KHz}, 2 \mathrm{~V}, 60 \%$ respectively. At the same time, two signals need to be compared using voltage comparator. One of the signals is the desired signal (reference signal which is taken from the BJT collector terminal) and the other is the controlled signal (input sensed voltage). The output of the voltage comparator is either high " 1 " if the controlled signal is greater than the desired signal or low "0" when the controlled signal is less than the desired signal. Thus, the output of the voltage comparator will determine the new duty cycle of the PWM signal. Finally, the AND gate is used to match the output of the voltage comparator and PWM signal generated from the function 
generator. As a result, the output signal from the AND gate will be a PWM signal with a different duty cycle depending on the value of the desired signal and the controlled signal. Note that in effect, it is somewhat similar to burst mode control technique.

\section{EXPERIMENTAL SETUP}

Figs. 7 and 8 show a block diagram of the implemented system and the schematic diagram respectively. The main competent for the proposed circuit are DC-DC boost converter, BJT, voltage comparator, PWM signal generator and AND gate.

The feedback loop consists of a BJT transistor, a voltage comparator and a logic AND gate. The BJT transistor is to implement a variable reference voltage. The base terminal is connected to the output terminal of the boost converter, and the collector terminal is connected with a voltage source which is equal the voltage at the maximum power $\left(\mathrm{V}_{\mathrm{mpp}}\right)$ while the emitter is grounded. As a result, the reference collector voltage will change depending on the changes in

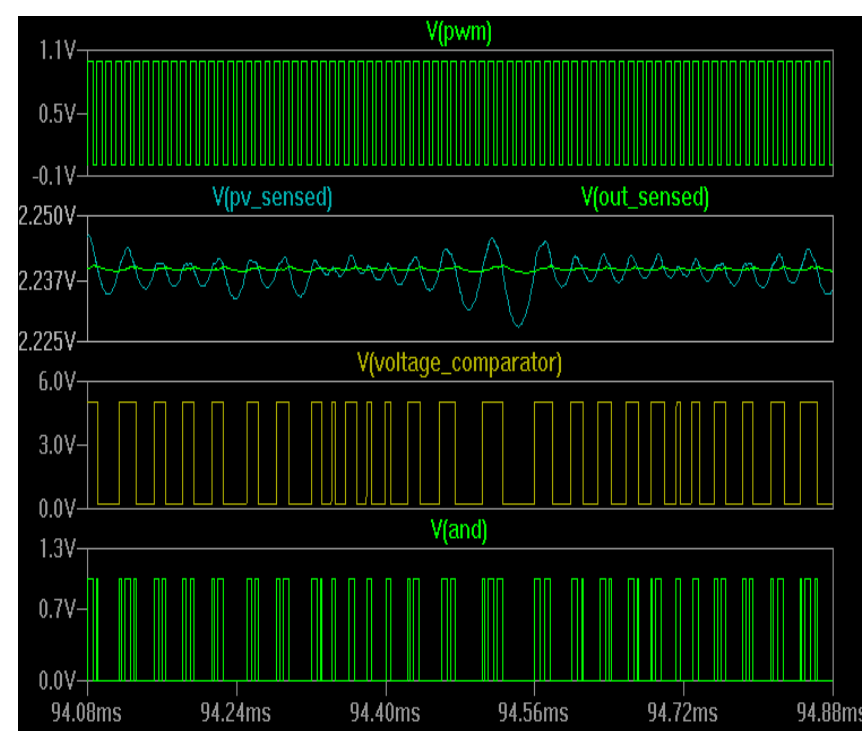

Fig. 6. PWM signal genrated in analoge control.

the base voltage. Thus, any changes in the radiation will lead to a change in the reference voltage without a need to use a digital control and a PID controller.

The function of the voltage comparator is to compare the input and the output voltage. If the PV operation voltage less than the reference voltage the output voltage of the voltage comparator will be high " 1 " otherwise it will be low "0". The output of the comparator will be the input of the AND gate. The AND gate used to control the duty cycle of the PWM signal and it has two terminals, the first one from the voltage comparator and the second from the function generator that generates PWM signal in constant frequency and duty cycle. Note that the maximum duty cycle is set by duty cycle generator so deadtime of duty cycle can be adjusted and $100 \%$ duty cycle can be avoided. By conducting and dis-conducting the AND gate, the PWM signal will be reshaped to the proper PWM signal with a different duty cycle.

The output terminal of the AND gate connect to the gate of the MOSFET switch of boost converter. Because the duty cycle is changed the boost converter will match the input and output impedance and the maximum power point will be tracking

A $20 \mathrm{~W}$ photovoltaic panel was used, and the experiment was contacted in the laboratory where the PV module was not being exposed to lighting from the outdoor, so one 500W halogen lamp and two $30 \mathrm{~W}$ led lamp were used to simulate different solar radiation levels. The first stage in the experiment was to get the I-V and P-V curves of the PV panel at different solar radiation levels, so the PV panel was connected with variable resistive bank. Its value varies between 0 to 250 ohms. Thus, the PV panel voltage and current were recorded.

The second stage was to verify the proposed circuit, so the proposed circuit was built and tested at different solar radiations as shown in Fig. 9. The halogen lamp (500 watt) was used to act as a full solar radiation while two LED lamp (30 watt each) was used to act as if it was cloudy weather. The distance between the PV module and the lamp was continually changed to get different solar insulation in both case. All parameters for experiment are listed in Table I. 


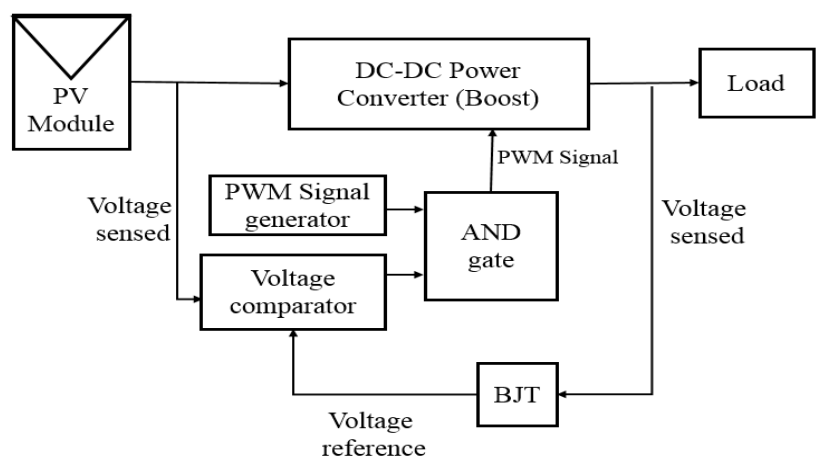

Fig. 7. Proposed circuit.

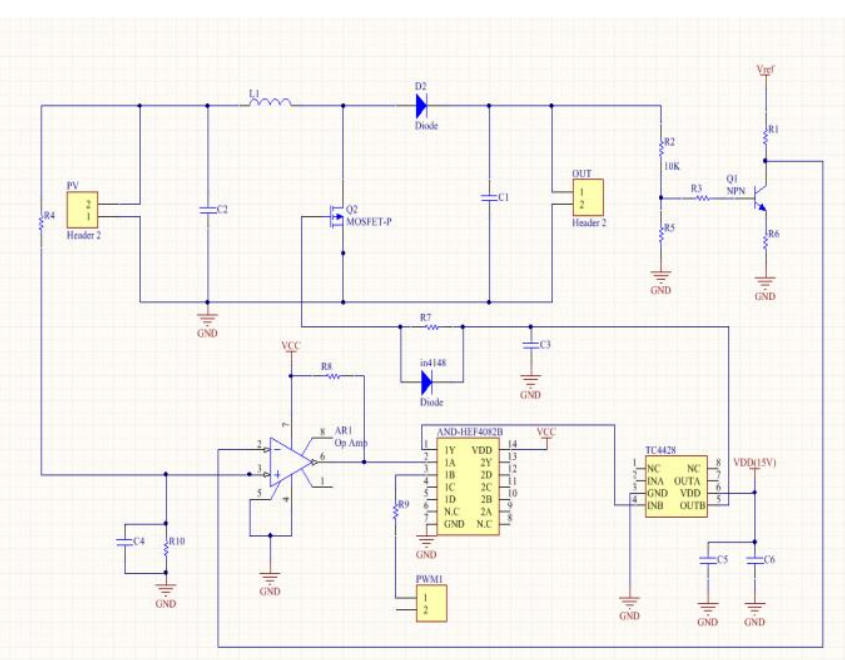

Fig. 8. Schematic diagram of the experimental setup.

TABLE I. PARAMETERS OF THE EXPERIMENTAL SYSTEM.

\begin{tabular}{|l|l|l|l|}
\hline C1 & $470 \mu \mathrm{F}$ & AND & HEF4082B \\
\hline C2 & $100 \mu \mathrm{F}$ & Op-Amp & LT1011 \\
\hline C3 & $1000 \mathrm{PF}$ & BJT & BC549 \\
\hline C4 & $0.1 \mu \mathrm{F}$ & R1 & $720 \Omega$ \\
\hline C5 & $0.1 \mu \mathrm{F}$ & R2 & $10 \mathrm{~K} \Omega$ \\
\hline C6 & $4.7 \mu \mathrm{F}$ & R3 & $600 \Omega$ \\
\hline L1 & $100 \mu \mathrm{H}$ & R4 & $9 \mathrm{~K} \Omega$ \\
\hline Q2 & IRF540N & R5,R7,R8 & $1 \mathrm{~K} \Omega$ \\
\hline D2 & BY229 & R6 & $2 \mathrm{~K} \Omega$ \\
\hline
\end{tabular}




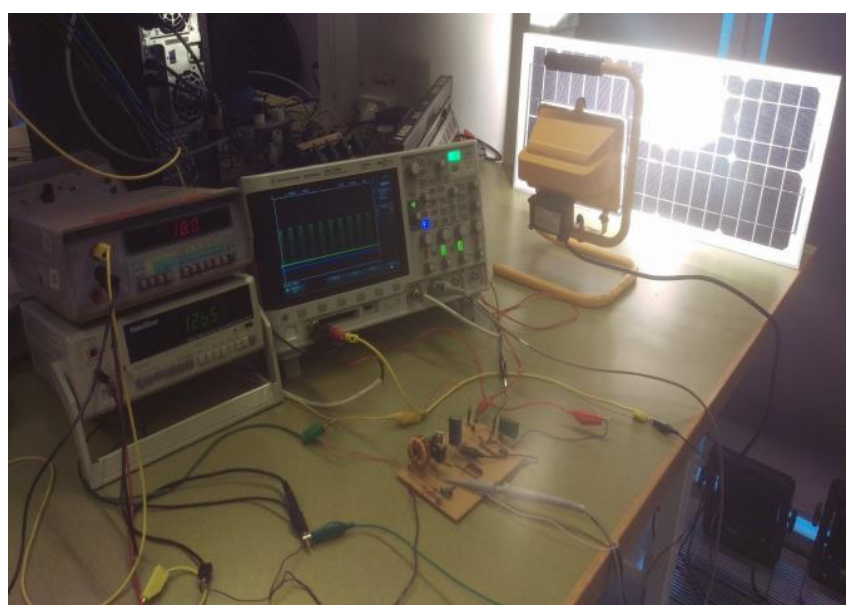

Fig. 9. Laboratory experimentation.

\section{EXPERIMENTAL RESULTS}

PV panel characteristic I-V and P-V curves are shown in Figs. 10 and 11 respectively. These curves are generated by connecting the PV panel to a variable resistor load under different solar radiation (radiation from Halogen and LED lamp). Voltage and current were measured for each resistive and radiation value.

The reference voltage at the maximum power point was found to be $17.9 \mathrm{~V}$ which is close to the voltage at the maximum power point $\left(\mathrm{V}_{\mathrm{MPP}}\right)$ that is given in the PV panel datasheet which is equal 18V. However, when less radiation occurred such as the

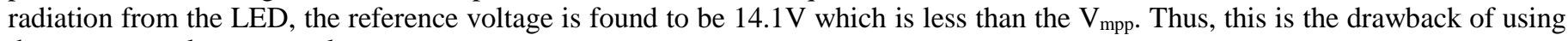
the constant voltage control.

However, in the proposed algorithm this difference in the reference voltage was considered by using simple and cost effective feedback circuit. The main component of this feedback loop is BJT transistor. As a result, the reference voltage will change according the change in the solar radiation. This change can lead to true maximum power point if the characteristic of the BJT was seated probably. The PV panel voltage and the output voltage for the proposed algorithm is shown in Fig.12.

The experiment result shows improvement in the time the needed to track MPP. It is required $150 \mathrm{~ms}$ to track the maximum power and that will reduce the power loss during MPP tracking.

As this method is designed according the characteristic of the PV module, it is recommended to use this method with each PV module in the system to avoid the effect of the partial shading If it is required to use more than one PV module, However, this circuit could be added to each PV module as it is cost effective.

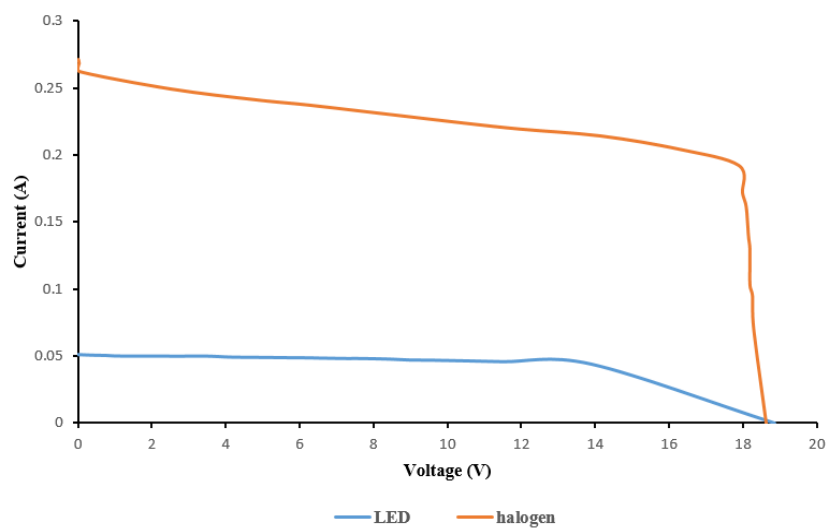

Fig. 10. I-V curve for PV Panel at different radiation. 


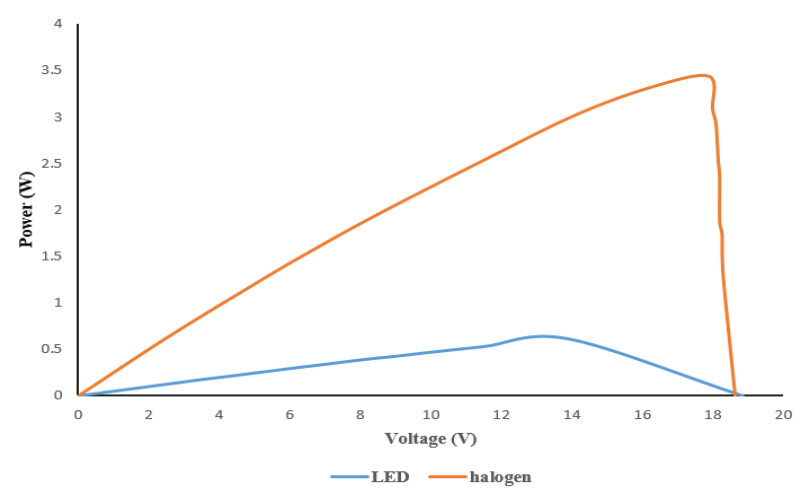

Fig. 11. P-V curve for PV Panel at different radiation.

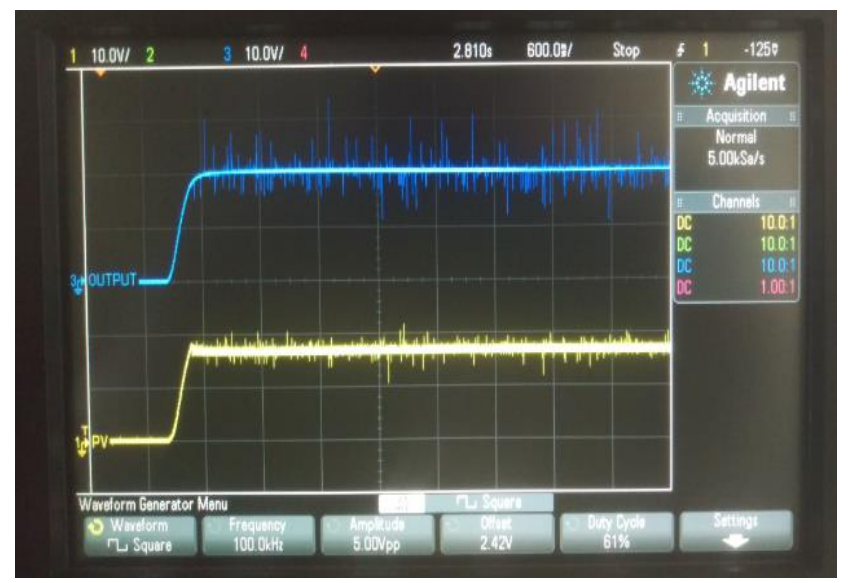

Fig. 12. PV and output voltage vs time.

\section{CONCLUTION}

This paper presents an improved analog maximum power point tracking algorithm for PV system. The proposed circuit is based on simple voltage feedforward control. In addition, it takes the advantage of BJT transistor where the collector current is used to mimic a voltage slope which connects the maximum power voltage points for more accurate tracking.

\section{References}

[1] E. Koutroulis, K. Kalaitzakis, and N. C. Voulgaris, "Development of a microcontroller-based, photovoltaic maximum power point tracking control system," IEEE Transactions on Power Electronics, vol. 16, pp. 46-54, 2001.

[2] A. Bidram, A. Davoudi, and R. S. Balog, "Control and Circuit Techniques to Mitigate Partial Shading Effects in Photovoltaic Arrays," IEEE Journal of Photovoltaics, vol. 2, pp. 532-546, 2012.

[3] A. Pandey, N. Dasgupta, and A. K. Mukerjee, "High-Performance Algorithms for Drift Avoidance and Fast Tracking in Solar MPPT System," IEEE Transactions on Energy Conversion, vol. 23, pp. 681-689, 2008.

[4] V. Salas, E. Olias, A. Barrado, and A. Lazaro, "Review of the maximum power point tracking algorithms for stand-alone photovoltaic systems," Solar energy materials and solar cells, vol. 90, pp. 1555-1578, 2006.

[5] B. Subudhi and R. Pradhan, "A Comparative Study on Maximum Power Point Tracking Techniques for Photovoltaic Power Systems," IEEE Transactions on Sustainable Energy, vol. 4, pp. 89-98, 2013.

[6] J. Ahmad, "A fractional open circuit voltage based maximum power point tracker for photovoltaic arrays," in Software Technology and Engineering (ICSTE), 2010 2nd International Conference on, 2010, pp. V1-247-V1-250.

[7] M. R. Al-Soeidat, A. Cembrano, and D. D. C. Lu, "Comparing effectiveness of hybrid mppt algorithms under partial shading conditions," in 2016 IEEE International Conference on Power System Technology (POWERCON), 2016, pp. 16. 
[8] H. Chihchiang and S. Chihming, "Comparative study of peak power tracking techniques for solar storage system," in Applied Power Electronics Conference and Exposition, 1998. APEC '98. Conference Proceedings 1998., Thirteenth Annual, 1998, pp. 679-685 vol.2.

[9] N. Mohan and T. M. Undeland, Power electronics: converters, applications, and design: John Wiley \& Sons, 2007. 